\title{
Impact of theoretical assumptions in the determination of the neutrino effective number from future CMB measurements
}

Dol:

10.1103/PhysRevD.97.063519

\section{Document Version}

Final published version

Link to publication record in Manchester Research Explorer

Citation for published version (APA):

Capparelli, L., Di Valentino, E., Melchiorri, A., \& Chluba, J. (2018). Impact of theoretical assumptions in the determination of the neutrino effective number from future CMB measurements. Physical Review D, 97(6), [063519]. https://doi.org/10.1103/PhysRevD.97.063519

\section{Published in:}

Physical Review D

\section{Citing this paper}

Please note that where the full-text provided on Manchester Research Explorer is the Author Accepted Manuscript or Proof version this may differ from the final Published version. If citing, it is advised that you check and use the publisher's definitive version.

\section{General rights}

Copyright and moral rights for the publications made accessible in the Research Explorer are retained by the authors and/or other copyright owners and it is a condition of accessing publications that users recognise and abide by the legal requirements associated with these rights.

\section{Takedown policy}

If you believe that this document breaches copyright please refer to the University of Manchester's Takedown Procedures [http://man.ac.uk/04Y6Bo] or contact uml.scholarlycommunications@manchester.ac.uk providing relevant details, so we can investigate your claim.

\section{OPEN ACCESS}




\title{
Impact of theoretical assumptions in the determination of the neutrino effective number from future CMB measurements
}

\author{
Ludovico Capparelli, ${ }^{1, *}$ Eleonora Di Valentino, ${ }^{2, \dagger}$ Alessandro Melchiorri, ${ }^{1, *}$ and Jens Chluba ${ }^{2, \S}$ \\ ${ }^{1}$ Physics Department and INFN, Università di Roma "La Sapienza", Ple Aldo Moro 2, 00185 Rome, Italy \\ ${ }^{2}$ Jodrell Bank Center for Astrophysics, School of Physics and Astronomy, University of Manchester, \\ Oxford Road, Manchester M13 9PL, United Kingdom
}

(Received 10 January 2018; published 19 March 2018)

\begin{abstract}
One of the major goals of future cosmic microwave background (CMB) measurements is the accurate determination of the effective number of neutrinos $N_{\text {eff }}$. Reaching an experimental sensitivity of $\Delta N_{\text {eff }}=0.013$ could indeed falsify the presence of any nonstandard relativistic particles at $95 \%$ C.L. In this paper, we test how this future constraint can be affected by the removal of two common assumptions: a negligible running of the inflationary spectral index $n_{\text {run }}$ and a precise determination of the neutron lifetime $\tau_{n}$. We first show that the constraints on $N_{\text {eff }}$ could be significantly biased by the unaccounted presence of a running of the spectral index. Considering the Stage-IV experiment, a negative running of $\mathrm{d} n / \mathrm{d} \ln k=-0.002$ could mimic a positive variation of $\Delta N_{\text {eff }}=0.03$. Moreover, given the current discrepancies between experimental measurements of the neutron lifetime $\tau_{n}$, we show that the assumption of a conservative error of $\Delta \tau_{n} \sim 10 \mathrm{~s}$ could cause a systematic error of $\Delta N_{\text {eff }}=0.02$. Complementary cosmological constraints on the running of the spectral index and a solution to the neutron lifetime discrepancy are therefore needed for an accurate and reliable future CMB bound of $N_{\text {eff }}$ at the percent level.
\end{abstract}

DOI: $10.1103 /$ PhysRevD.97.063519

\section{INTRODUCTION}

In the past decade, measurements of the cosmic microwave background (CMB) anisotropies made from satellite experiments such as the Wilkinson Microwave Anisotropy Probe (WMAP) [1] and Planck [2] have spectacularly confirmed the theoretical expectations of the standard model of structure formation based on inflation, cold dark matter, and a cosmological constant. This nearly perfect agreement between theory and observations is now letting cosmologists use the CMB data to constrain several aspects of fundamental physics (see e.g. [2]).

Among these parameters, a key observable is the effective neutrino number, $N_{\text {eff }}$, that determines the number of relativistic degrees of freedom during the epoch of CMB anisotropies formation, at the last scattering surface (see e.g. [3]). A change in the neutrino effective number affects the epoch of equality and modifies the CMB horizon and damping scales, yielding a characteristic imprint on the CMB [4]. The latest measurement made by the Planck satellite [2,5] constrains this parameter to $N_{\text {eff }}=2.91_{-0.37}^{+0.39}$ at $95 \%$ C.L. in agreement with the standard model

\footnotetext{
*ludovico.mark.capparelli@roma1.infn.it †eleonora.divalentino@manchester.ac.uk *alessandro.melchiorri@roma1.infn.it

§jens.chluba@manchester.ac.uk
}

expectation of $N_{\text {eff }}=3.046$ (corresponding to three active neutrinos) and with a $\sigma\left(N_{\text {eff }}\right) \sim 0.2$ accuracy.

While currently there is no observational indication for a nonstandard value of $N_{\text {eff }}$, there are several physical mechanisms that can change its value. Sterile neutrinos [6], gravitational waves [7], axions [8], gravitino decays [9], and self-interacting dark matter [10] (just to name a few) can indeed all modify $N_{\text {eff }}$.

As discussed in [11], any particle that decouples from the primordial thermal plasma before the QCD transition will contribute with $\Delta N_{\text {eff }}=N_{\text {eff }}-3.046 \geq 0.3$. This number has already been tested with Planck, and near future data can fully falsify this hypothesis. If, however, the coupling happens after the QCD transition, then any relativistic particle will contribute with a minimum value of $\Delta N_{\text {eff }}=0.027$. More precisely, the minimum contribution for a single real scalar particle is $\Delta N_{\text {eff }}=0.027$, for a Weyl fermion it is $\Delta N_{\text {eff }}=0.047$, and for a light vector boson it is $\Delta N_{\text {eff }}=0.054$ [11]. It is therefore clear that future CMB experiments reaching an experimental sensitivity of $\sigma\left(N_{\text {eff }}\right)=0.013$ will have the potential to rule out the existence of any relativistic particle beyond those predicted in the standard model at more than $95 \%$ C.L.

Reaching an accuracy on $N_{\text {eff }}$ that is almost 15 times better than current uncertainties is clearly an ambitious and difficult task. In [12] (see also [13]) it has been shown that, in principle, $\mathrm{CMB}$ observations could reach this sensitivity, 
provided we have a perfect foreground removal, an angular resolution of $\sim 1^{\prime}$, a sampled sky fraction above $f_{\text {sky }}>0.6$, and a noise detector in temperature of $0.2 \mu \mathrm{K}$-arcmin (see Fig. 25 in [12]).

While the signal could indeed be present in the CMB sky, in this paper we highlight two key assumptions made in the forecasts that could undermine the possibility of reaching this sensitivity even for an ideal experiment.

The first assumption concerns inflation. In the forecasts made in [12], the power spectrum of primordial perturbations has been assumed to follow the usual power law form $P(k)=A_{S} k^{n_{S}}$, where $k$ is the perturbation comoving scale, and $A_{S}$ and $n_{S}$ are the inflationary scalar amplitude and spectral index, respectively (see e.g. [14]). However, inflation generally predicts the presence of a running spectral index $n_{\text {run }}=\mathrm{d} n_{S} / \mathrm{d} \ln k$ of the order of $\left(1-n_{S}\right)^{2} \sim 0.001$. Varying $n_{\text {run }}$ produces a similar effect in the CMB spectrum of a variation in the neutrino number. The inclusion of the running in the analysis introduces a degeneracy between $n_{\text {run }}$ and $N_{\text {eff }}$ that significantly weakens the achievable $\Delta N_{\text {eff }}$. Moreover, an unaccounted for negative running could mimic a value for $\Delta N_{\text {eff }}>0$, suggesting the presence of new light particles.

The second assumption we investigate is related to the value of the primordial helium abundance. It is well known that the helium abundance parameter $Y_{p}$ is strongly degenerate with $N_{\text {eff }}$ (see e.g. [4]). Letting $Y_{p}$ also vary freely in the analysis would greatly weaken the bounds on $N_{\text {eff. }}$. The most stringent forecasts presented in [12] or [13] assume a helium abundance derived from standard big bang nucleosynthesis (BBN). However, even the most accurate BBN code, given a value of the baryon density and $N_{\text {eff }}$, will produce an estimate of $Y_{p}$ that is affected by a small uncertainty. This uncertainty mainly comes from the current experimental error on the neutron lifetime $\tau_{n}$. According to the latest Particle Data Group (PDG) edition [15], the neutron lifetime is known with a precision of $\tau_{n}=$ $880.2 \pm 1.0 \mathrm{~s}$ at $68 \%$ C.L., but this is an averaged value over different experimental constraints that are discrepant at the level of 4 standard deviations. A larger, more conservative uncertainty on the value of $\tau_{n}$ can therefore affect the precision in $Y_{p}$ and the final accuracy in $\Delta N_{\text {eff }}$.

The goal of this paper is therefore to assess the impact of these two assumptions in future determinations of $N_{\text {eff }}$ from CMB anisotropies.

\section{METHOD}

In this paper, we forecast the ability of future $\mathrm{CMB}$ experiments to constrain the effective neutrino number $N_{\text {eff }}$ in different theoretical frameworks.

Following the now common approach already used, for example, in [13], we perform Markov chain Monte Carlo (MCMC) analyses on mock data for several possible future experimental configurations, assuming a fiducial, vanilla, flat $\Lambda \mathrm{CDM}$ model compatible with the recent Planck 2015 results [5]. More specifically, we assume baryon and cold dark matter densities of $\Omega_{b} h^{2}=0.02225$ and $\Omega_{c} h^{2}=0.1198$, respectively, an optical depth $\tau=0.0596$, an inflationary spectral index $n_{s}=0.9645$, and 3 neutrinos with effective number $N_{\text {eff }}=3.046$. While we consider $\Lambda \mathrm{CDM}$ as the fiducial model, in our MCMC analysis we also varied the neutrino effective number $N_{\text {eff }}$ and the running $n_{\text {run }}$.

We use the publicly available Boltzmann code, CAMB [16], to compute the theoretical $\mathrm{CMB}$ angular power spectra $C_{\ell}^{T T}, C_{\ell}^{T E}, C_{\ell}^{E E}$, and $C_{\ell}^{B B}$ for temperature, crosstemperature polarization, and $E$ and $B$ modes polarization. ${ }^{1}$

In our simulations we make use of an instrumental noise given by the usual expression

$$
N_{\ell}=w^{-1} \exp \left(\ell(\ell+1) \theta^{2} / 8 \ln 2\right),
$$

where $\theta$ is the experimental full-width-at-half-maximum (FWHM) angular resolution, and $w^{-1}$ is the raw experimental sensitivity expressed in $(\mu \mathrm{K}-\mathrm{rad})^{2}$. The total variance of the multipoles $a_{l m}$ is therefore given by the sum of the fiducial $C_{\ell}$ 's with the instrumental noise $N_{\ell}$.

We consider two experimental configurations: a Stage-IV experiment as in [17] and a futuristic/optimistic "Stage-IV+" configuration with improved angular resolution and sensitivity as suggested in [12]. We generate fiducial and noise spectra with noise properties as reported in Table I. Since we are mainly interested here in the impact of theoretical assumptions, we assume negligible beam uncertainties and no foreground contaminations. However, we limit the temperature and polarization power spectrum from Stage-IV data in the range $5 \leq \ell \leq 3000$. As we report in the next section with the Stage-IV configuration reported in Table I, we get forecasts on $N_{\text {eff }}$ with uncertainties that are about $\sim 20 \%$ larger than those reported in [12]. For the more optimistic Stage-IV+ configuration, we consider $\ell_{\max }=5000$. For both configurations we consider a sampled sky fraction of $f_{\text {sky }}=0.4$. We do not include simulated Planck data with $f_{\text {sky }}=0.2$ as in [12].

The simulated experimental data are then compared with a MCMC method with a theoretical model assuming a Gaussian likelihood $\mathcal{L}$ given by

$$
-2 \ln \mathcal{L}=\sum_{l}(2 l+1) f_{\text {sky }}\left(\frac{D}{|\bar{C}|}+\ln \frac{|\bar{C}|}{|\hat{C}|}-3\right)
$$

where $\bar{C}_{l}$ and $\hat{C}_{l}$ are the assumed fiducial and theoretical spectra plus noise, respectively, and $|\bar{C}|$ and $|\hat{C}|$ are the

\footnotetext{
${ }^{1}$ We neglect the non-Gaussianity of the lensed $B$ modes, and we do not delense. Our assumptions are therefore slightly more conservative than those presented in [12] where simulated Planck data were also considered.
} 
TABLE I. Experimental specifications for the two configurations considered in the forecasts.

\begin{tabular}{lccccc}
\hline \hline Configuration & Beam & Raw sensitivity $w^{-1 / 2}$ & $\ell_{\max }$ & $\ell_{\min }$ & $f_{\text {sky }}$ \\
\hline Stage-IV & 3 & $1(\mu K$-arcmin $)$ & 3000 & 5 & 0.4 \\
Stage-IV+ & 1, & $0.5(\mu K$-arcmin $)$ & 5000 & 2 & 0.4 \\
\hline \hline
\end{tabular}

determinants of the theoretical and observed data covariance matrices given by

$$
\begin{aligned}
& |\bar{C}|=\bar{C}_{\ell}^{T T} \bar{C}_{\ell}^{E E} \bar{C}_{\ell}^{B B}-\left(\bar{C}_{\ell}^{T E}\right)^{2} \bar{C}_{\ell}^{B B}, \\
& |\hat{C}|=\hat{C}_{\ell}^{T T} \hat{C}_{\ell}^{E E} \hat{C}_{\ell}^{B B}-\left(\hat{C}_{\ell}^{T E}\right)^{2} \hat{C}_{\ell}^{B B},
\end{aligned}
$$

where $D$ is

$$
\begin{aligned}
D= & \hat{C}_{\ell}^{T T} \bar{C}_{\ell}^{E E} \bar{C}_{\ell}^{B B}+\bar{C}_{\ell}^{T T} \hat{C}_{\ell}^{E E} \bar{C}_{\ell}^{B B}+\bar{C}_{\ell}^{T T} \bar{C}_{\ell}^{E E} \hat{C}_{\ell}^{B B} \\
& -\bar{C}_{\ell}^{T E}\left(\bar{C}_{\ell}^{T E} \hat{C}_{\ell}^{B B}+2 \hat{C}_{\ell}^{T E} \bar{C}_{\ell}^{B B}\right) .
\end{aligned}
$$

For our MCMC runs we use the publicly available Markov chain Monte Carlo package CosmoMC ${ }^{2}$ [18], sampling parameters with the Metropolis-Hastings algorithm, with a convergence diagnostic based on the Gelman and Rubin statistic.

\section{RESULTS}

In this section we present our findings, discussing the impact of the two mentioned assumptions in the determination of $N_{\text {eff }}$.

\section{A. Impact of the running of the spectral index}

We first analyze the case of a possible presence of running of the inflationary spectral index. We remind the reader that the slow-roll solution for the primordial power spectrum can be expressed as (see e.g. [19])

$$
\begin{aligned}
1-n_{\mathrm{s}} & =2 \epsilon-\frac{\epsilon_{, N}}{\epsilon}-\frac{c_{\mathrm{s}, N}}{c_{\mathrm{s}}} \\
& =\frac{r}{8 c_{\mathrm{s}}}-\frac{r_{, N}}{r}, \\
n_{\mathrm{run}} & =2 \epsilon_{, N}-\frac{r_{, N N}}{r}+\left(\frac{r_{, N}}{r}\right)^{2},
\end{aligned}
$$

where $\epsilon$ is the slow-roll parameter, $c_{\mathrm{s}}$ is the inflaton speed of sound, , $N$ refers to a derivative with respect to the number of e-foldings (see e.g. [14]), and the tensor-toscalar ratio is given approximately by $r=16 \epsilon c_{\mathrm{s}}$.

\footnotetext{
${ }^{2}$ See http://cosmologist.info.
}

Combining the above equations, it is possible to write

$$
n_{\text {run }}=\left(1-n_{\mathrm{s}}\right)^{2}-6 \epsilon\left(1-n_{\mathrm{s}}\right)+8 \epsilon^{2}-\left(\frac{r c_{\mathrm{s}, \mathrm{N}}}{8 c_{\mathrm{s}}^{2}}+\frac{r_{, N N}}{r}\right) \text {. }
$$

In a typical slow-roll model, $n_{\text {run }}$ is therefore naturally expected to be of the same order of $\left(1-n_{\mathrm{s}}\right)^{2}$. Assuming a value of $n_{s}=0.955$, compatible in between 2 standard deviations with current constraints from Planck [2], we have $\left(1-n_{\mathrm{s}}\right)^{2} \sim 0.002$, which is approximately the same level of expected sensitivity on $n_{\text {run }}$ for the Stage-IV experiment [12].

More specifically, if we consider the Starobinsky model [20] with $c_{\mathrm{s}}=1$ and $\epsilon=3 /\left(4 N^{2}\right)$, we obtain

$$
\begin{gathered}
\left(1-n_{\mathrm{s}}\right)^{2} \simeq \frac{4}{N^{2}}, \\
\frac{r_{, N N}}{r}=\frac{6}{N^{2}},
\end{gathered}
$$

which corresponds to a value for the running (again for $\left.n_{s}=0.955\right)$ of

$$
n_{\text {run }} \simeq-\frac{2}{N^{2}} \simeq-\frac{1}{2}\left(1-n_{\mathrm{s}}\right)^{2} \simeq-0.001 .
$$

It will not be possible for Stage-IV alone to detect the running in the case of the Starobinsky model, and several other models predict a similar running (e.g. see [21]). However, as we discuss below, if not considered in the analysis it may still affect the constraints on other, correlated, parameters such as $N_{\text {eff }}$. Moreover, a larger running is expected in several theoretical scenarios such as (just to name a few) a breakdown of the slow-roll approximation [22], multiple-field inflation [23], the presence of a noncanonical kinetic term [24], and the runningmass models [25].

Note that we adopt the commonly used parametrization for primordial power spectrum, truncating at $n_{\text {run }}$ (see e.g. $[1,2])$. Clearly this truncation is incomplete at describing models with large running $\left(n_{\text {run }} \sim 0.01\right)$ and that therefore significantly deviate from slow roll. Alternative parametrizations, which use a different prescription for resumming higher order terms, are available [26,27]. Similarly, extended parametrizations (e.g. with running as in [28] or with even larger numbers of parameters as in [29]) could be considered. However, a detailed assessment of the physical and observational differences for these extensions is beyond the scope of this work.

It is therefore interesting to perform an analysis on future mock data allowing both $N_{\text {eff }}$ and $n_{\text {run }}$ to vary. The results of this analysis are reported in Table II and in Figs. 1 and 2. As we can see from Fig. 1 and from the first row of Table II, a strong degeneracy between the running of the spectral index and the neutrino effective number exists. Namely, 
TABLE II. Constraints at $68 \%$ C.L. for $N_{\text {eff }}$ assuming different values for the running. Including the running in the analysis (first row) increases the error on $N_{\text {eff }}$ by $\sim 76 \%$ for the Stage-IV experiment $(\sim 17 \%$ for a Stage-IV+ experiment) with respect to the no-running case (second row). Neglecting the running shifts the mean value by approximately $\Delta N_{\text {eff }} \sim-12 n_{\text {run }}$ for Stage-IV and $\Delta N_{\text {eff }} \sim-5 n_{\text {run }}$ for a Stage-IV+ experiment.

\begin{tabular}{lcc}
\hline \hline Case & $N_{\text {eff }}($ Stage-IV $)$ & $N_{\text {eff }}($ Stage-IV +$)$ \\
\hline Varying $n_{\text {run }}$ & $3.049 \pm 0.076$ & $3.048_{-0.026}^{+0.023}$ \\
$n_{\text {run }}=0$ & $3.048 \pm 0.043$ & $3.047 \pm 0.021$ \\
$n_{\text {run }}=0.002$ & $3.019 \pm 0.043$ & $3.035 \pm 0.021$ \\
$n_{\text {run }}=0.004$ & $2.996 \pm 0.044$ & $3.024 \pm 0.021$ \\
$n_{\text {run }}=-0.002$ & $3.074 \pm 0.044$ & $3.056 \pm 0.021$ \\
$n_{\text {run }}=-0.004$ & $3.098 \pm 0.044$ & $3.071 \pm 0.019$ \\
\hline \hline
\end{tabular}

a decrease/increase of $N_{\text {eff }}$ can be counterbalanced with a decrease/increase of $n_{\text {run }}$. The main effect of this degeneracy is a significant increase in the forecasted uncertainty on $N_{\text {eff }}$ when the $n_{\text {run }}$ parameter is considered. Indeed, by comparing the constraints between the first two rows of Table II we see that the inclusion of $n_{\text {run }}$ could result in an $\sim 76 \%$ decrease in the accuracy on $N_{\text {eff }}$ for a Stage-IV experimental configuration. In practice, opening the natural possibility of a running prevents the Stage-IV experiment from reaching the goal of a $\sigma\left(N_{\text {eff }}\right) \sim 0.03$ accuracy. The $n_{\text {run }}-N_{\text {eff }}$ degeneracy is less present in the case of a Stage-IV+ experiment. Still, when running is considered, the constraints on $N_{\text {eff }}$ are weaker also in this case.

It is interesting to investigate what effect on $N_{\text {eff }}$ could be produced by unaccounted running, i.e., when performing a

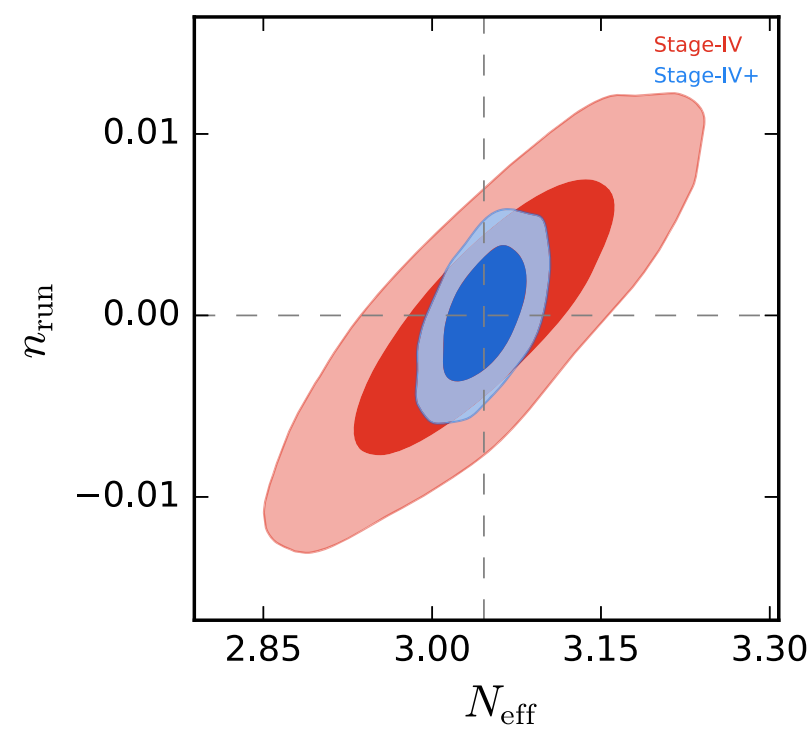

FIG. 1. Contour plots at the $68 \%$ and $95 \%$ confidence level forecasted on the $n_{\text {run }}$ vs $N_{\text {eff }}$ plane for the Stage-IV experiment and for an optimistic Stage-IV+ upgrade. A degeneracy between the two parameters is clearly present and is more pronounced in the case of Stage-IV.
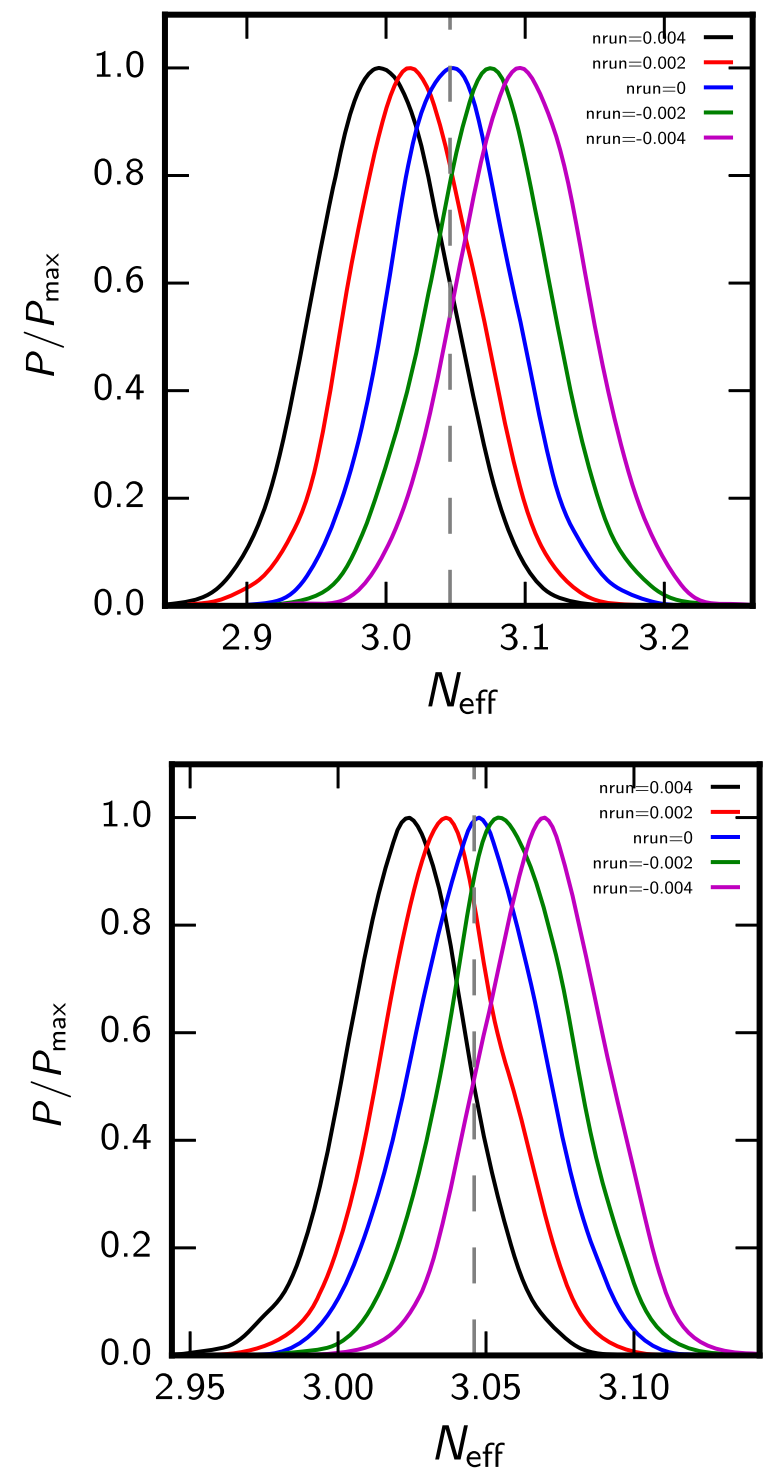

FIG. 2. Posterior distributions on $N_{\text {eff }}$ assuming $N_{\text {eff }}=3.046$ and different values for $n_{\text {run }}$ for the fiducial model but performing an analysis with $n_{\text {run }}=0$. In the top panel are the posteriors from Stage-IV, while in the bottom we have the posteriors for a Stage-IV+ experiment. As we can see, not accounting for a negative running could produce a significant shift in the recovered values of $N_{\text {eff }}$.

MCMC analysis by fixing $n_{\text {run }}=0$ but adding nonzero running to the fiducial model. As we can see from Table II and the posteriors in Fig. 2, we found that unaccounted running could shift the mean value of $N_{\text {eff }}$ from the standard value $N_{\text {eff }}=3.046$ by

$$
\Delta N_{\text {eff }} \sim-12 n_{\text {run }}
$$

for a Stage-IV configuration and

$$
\Delta N_{\text {eff }} \sim-5 n_{\text {run }}
$$


for a Stage-IV+ experiment. As we can see, therefore, even a small negative running of $n_{\text {run }} \sim-0.001$ could produce a positive shift of $\Delta N_{\text {eff }} \sim 0.01$ in the recovered mean value of $N_{\text {eff }}$ for Stage-IV. Moreover, one should consider that Stage-IV will have an accuracy of $\sigma\left(n_{\text {run }}\right) \sim 0.002$ at $68 \%$ C.L. Even with a value of $n_{\text {run }}=0.001$, a statistical fluctuation of $\sim 1.5$ standard deviation could be possible, yielding $n_{\text {run }} \sim 0.004$ and a shift of $\Delta N_{\text {eff }} \sim 0.048$. In practice, it will be hard for Stage-IV to discriminate between a negative running and the presence of a light vector boson.

Fortunately, the situation appears brighter when considering a Stage-IV+ experiment. In this case, as we can see from Figs. 1 and 2, bottom panel, the degeneracy between $N_{\text {eff }}$ and $n_{\text {run }}$ is less significant. Without running, we find that a Stage-IV+ experiment could reach a sensitivity of $\Delta N_{\text {eff }}=0.021$ (see Table II), excluding at more than $95 \%$ C.L. the minimum contribution of $\Delta N$ eff $=0.054$ for a light vector boson. However, in this case a positive running of $n_{\text {run }} \sim 0.001$ could produce a negative shift of $\Delta N_{\text {eff }} \sim-0.005$. This would be enough for bringing such a signal back in agreement between the 2 standard deviation threshold.

\section{B. Impact of the neutron lifetime}

The second key assumption we want to investigate concerns the value of the neutron lifetime. The forecasts on $N_{\text {eff }}$ presented in [12] and in the previous section generally assume a value on the primordial helium abundance derived from BBN. In practice, for each theoretical model, a value for $Y_{p}$ is obtained through a BBN code ("Parthenope" [30]) assuming the baryon density $\omega_{b}$ of the model and a neutron lifetime of $\tau_{n}=880.3 \mathrm{~s}$. An accurate determination of the helium abundance $Y_{p}$ is crucial for the determination of $N_{\text {eff }}$. The two parameters are indeed correlated, and without the assumption of $\mathrm{BBN}$, the accuracy on $N_{\text {eff }}$ is larger than $\sigma\left(N_{\text {eff }}\right)=0.07$ even for the most optimistic experimental configuration (see [12]). However, the neutron lifetime is known with an experimental error. As it is well known, this uncertainty propagates in a systematic error on the $\mathrm{BBN}$ derived value of $Y_{p}$ that may affect the constraints on $N_{\text {eff }}$. Given a value of the neutron lifetime, one could expect from a numerical fit a helium abundance given by

$$
Y_{p}\left(\tau_{n}\right)=\left(\frac{\tau_{n}}{880.3 s}\right)^{0.73} Y_{p}\left(\tau_{n}=880.3 s\right),
$$

where $Y_{p}\left(\tau_{n}=880.3 s\right)$ is the helium abundance derived assuming $\tau_{n}=880.3 \mathrm{~s}$.

The most recent bound on the neutron lifetime from the PDG is [15]

$$
\tau_{n}=(880.2 \pm 1.0) s
$$

Assuming therefore a 2 standard deviation fluctuation, we could expect from Eq. (15) an increase in $Y_{p}$ of $\sim 0.18 \%$. This is about $\sim 15 \%$ of the expected Stage-IV accuracy on $Y_{p}$ of $\sigma\left(Y_{p}\right)=0.025$ (for $Y_{p}=0.2466$ ), and therefore it does not significantly affect the Stage-IV future constraint on $N_{\text {eff }}$.

However, there is a long-standing tension between different measurements of the neutron lifetime based on different experimental techniques (see e.g. [31-33]). The most recent measurements of the neutron lifetime are indeed based on two different experimental techniques: counting the $\beta$-decay products in a passing beam of cold neutrons ("beam" method) or counting the ultracold neutrons surviving in a storage bottle ("bottle" method).

The most recent value from the beam method is [34]

$$
\tau_{n}^{\text {beam }}=(887.7 \pm 1.2[\text { Stat }] \pm 1.9[\text { Sys }]) s,
$$

while the most precise measurement using the bottle method is [35]

$$
\tau_{n}^{\text {bottle }}=(878.5 \pm 0.7[\text { Stat }] \pm 0.3[\text { Sys }]) s .
$$

These two measurements (summing the systematic errors in quadrature) are therefore discrepant at the level of $\sim 3.9$ standard deviations. Moreover, the beam determination is in tension at the level of $\sim 3$ standard deviations with the quoted PDG constraint, while the bottle constraint also shows some tension, albeit just at 1.4 standard deviations.

Recently, new measurements with an improved bottle method have been reported [36], giving

$$
\tau_{n}^{\text {bottle }}=(877.7 \pm 0.7[\text { Stat }] \pm 0.3 /-0.1[\text { Sys }]) s,
$$

in tension with the PDG value at $\sim 2$ standard deviations.

Given the large inconsistencies between experimental values, it certainly makes sense to investigate how a larger uncertainty on $\tau_{n}$ than the one quoted in the PDG could impact future CMB constraints on $N_{\text {eff }}$.

We therefore simulate future $\mathrm{CMB}$ data assuming standard $\Lambda \mathrm{CDM}$ but with two possible "real" values for the neutron lifetime: $\tau_{n}^{\text {high }}=888.0 \mathrm{~s}$, consistent with beam measurements, and $\tau_{n}^{\text {low }}=877.0 \mathrm{~s}$, consistent with bottle experiments, corresponding to different values for the BBN derived primordial helium abundance. We then analyze these data sets assuming the quoted PDG value of $\tau_{n}=880.2 \mathrm{~s}$, recovering the value of $N_{\text {eff }}$ and quantifying the possible bias introduced by a wrong assumption on $\tau_{n}$ (and on the primordial helium abundance $Y_{p}$ ).

The results are reported in Table III. As we can see, an unaccounted for higher value for the neutron lifetime $\tau_{n}^{\text {high }}$ could introduce a bias in the neutrino effective number of $\Delta N_{\text {eff }} \sim 0.015$, while a lower value $\tau_{n}^{\text {low }}$ could produce $\Delta N_{\text {eff }} \sim-0.009$. In both the Stage-IV and Stage-IV+ cases, this possible systematic will not affect the $N_{\text {eff }}$ constraint in 
TABLE III. Constraints at $68 \%$ C.L. for $N_{\text {eff }}$ assuming different values for the neutron lifetime.

\begin{tabular}{lcc}
\hline \hline Case & $N_{\text {eff }}($ Stage-IV) & $N_{\text {eff }}$ (Stage-IV +$)$ \\
\hline$\tau_{n}=880.2 \mathrm{~s}$ & $3.048 \pm 0.043$ & $3.047 \pm 0.021$ \\
$\tau_{n}=888.0 \mathrm{~s}$ & $3.062 \pm 0.040$ & $3.064 \pm 0.021$ \\
$\tau_{n}=877.0 \mathrm{~s}$ & $3.039 \pm 0.041$ & $3.037 \pm 0.020$ \\
\hline \hline
\end{tabular}

a significant way such as to mimic a detection at more than 2 standard deviations. However, it may make a statistical fluctuation more significant than what it actually is and, conversely, reduce the significance of a real discovery. In concreto, assuming a conservative experimental uncertainty on the neutron lifetime of $\sim 10 \mathrm{~s}$ introduces a systematic error of $\left|\Delta N_{\text {eff }}\right| \sim 0.02$, placing a serious limitation on the ultimate goal of $\sigma\left(N_{\text {eff }}\right)=0.013$.

\section{CONCLUSIONS}

In this paper, we have considered the impact of two theoretical assumptions in the forecasted accuracy on the neutrino effective number for future $\mathrm{CMB}$ experiments. As an illustration, we have considered two experiments: the future Stage-IV experiment and a further, optimistic upgrade to a Stage-IV+ experiment. The first assumption concerns the running of the inflationary spectral index, usually assumed as negligible. The second assumption is related to a perfect knowledge of the neutron lifetime. Neither assumption is particularly well motivated: slow-roll inflation predicts a running of the same order of the accuracy expected from future experiments, while an $\sim 4 \sigma$ tension between current experimental measurements of $\tau_{n}$ is present, potentially suggesting a significantly larger systematic error.

We found that for the Stage-IV experiment a running of $n_{\text {run }} \sim-0.002\left(n_{\text {run }} \sim-0.004\right)$ could result in a positive shift of $\Delta N_{\text {eff }} \sim 0.03\left(\Delta N_{\text {eff }} \sim 0.05\right)$. Running could therefore strongly impact the abilities of the Stage-IV experiment to significantly rule out or detect the presence of an extra relativistic particle at recombination. In the case of a highly optimistic Stage-IV+ experiment, the correlation between running and $N_{\text {eff }}$ is less significant, and the results are less affected.

When considering the neutron lifetime, we found that if we assume the current uncertainties reported in the PDG then the impact is minimal. However, in the case of a different, larger value for $\tau_{n}$, compatible with current beam measurements, or smaller, compatible with the most recent bottle experiments, we found a shift of $\Delta N_{\text {eff }} \sim 0.016$ and $\Delta N_{\text {eff }} \sim-0.008$ for both Stage-IV and Stage-IV+. While we are clearly considering a very conservative uncertainty on $\tau_{n}$, nearly 10 times larger than that reported in the PDG, we also have to bear in mind that any claim of new physics from the CMB must withstand a severe scrutiny of the assumptions made.

Both running and current experimental uncertainties on $\tau_{n}$ can therefore undermine the possibility of reaching the accuracy of $\Delta N_{\text {eff }} \sim 0.013$ needed for ruling out the presence of any extra relativistic particle at more than 95\% C.L. Moreover, current uncertainties on the value of the neutron lifetime also limit the accuracy on $n_{\text {run }}$ achievable from future $\mathrm{CMB}$ experiments.

However, complementary cosmological observables such as galaxy surveys (see e.g. [37]), $21 \mathrm{~cm}$ line fluctuations [38,39], and, possibly, CMB spectral distortions (see e.g. $[19,40])$ could help in breaking the degeneracy between $n_{\text {run }}$ and $N_{\text {eff }}$. At the same time, new experiments expected in the next few years will be crucial in solving the current neutron lifetime discrepancy [41].

Before concluding, we want to point out that in this paper we discussed just two possible assumptions that can bias the derived value for $N_{\text {eff }}$. Other extensions of the standard model can produce similar effects. We plan to further analyze these extensions in a future paper [42]. We also confirmed that the remaining theoretical uncertainties between different standard recombination codes (e.g. CosmoRec [43] and Recfast [44,45]) produce effects that are below what was found here, even when including refined helium recombination physics [46].

\section{ACKNOWLEDGMENTS}

E. D. V. acknowledges support from the European Research Council in the form of Consolidator Grant No. 681431. A. M. thanks the University of Manchester and the Jodrell Bank Center for Astrophysics for hospitality. J. C. is supported by the Royal Society as a Royal Society University Research Fellow at the University of Manchester and the European Research Council through Consolidator Grant No. 725456, UK.
[1] G. Hinshaw et al. (WMAP Collaboration), Astrophys. J. Suppl. Ser. 208, 19 (2013).

[2] P. A. R. Ade et al. (Planck Collaboration), Astron. Astrophys. 594, A13 (2016).
[3] M. Archidiacono, E. Calabrese, and A. Melchiorri, Phys. Rev. D 84, 123008 (2011).

[4] Z. Hou, R. Keisler, L. Knox, M. Millea, and C. Reichardt, Phys. Rev. D 87, 083008 (2013). 
[5] N. Aghanim et al. (Planck Collaboration), Astron. Astrophys. 596, A107 (2016).

[6] S. Gariazzo, C. Giunti, M. Laveder, Y. F. Li, and E. M. Zavanin, J. Phys. G 43, 033001 (2016).

[7] T. L. Smith, E. Pierpaoli, and M. Kamionkowski, Phys. Rev. Lett. 97, 021301 (2006).

[8] E. Di Valentino, E. Giusarma, M. Lattanzi, O. Mena, A. Melchiorri, and J. Silk, Phys. Lett. B 752, 182 (2016).

[9] E. Dimastrogiovanni and L. M. Krauss, arXiv:1706.01495.

[10] R. Huo, M. Kaplinghat, Z. Pan, and H. B. Yu, arXiv: 1709.09717.

[11] D. Baumann, D. Green, and B. Wallisch, Phys. Rev. Lett. 117, 171301 (2016).

[12] K. N. Abazajian et al. (CMB-S4 Collaboration), arXiv: 1610.02743 .

[13] E. Di Valentino et al. (CORE Collaboration), arXiv: 1612.00021.

[14] D. Baumann, arXiv:0907.5424.

[15] C. Patrignani et al. (Particle Data Group), Chin. Phys. C 40, 100001 (2016) and 2017 update.

[16] A. Lewis, A. Challinor, and A. Lasenby, Astrophys. J. 538, 473 (2000).

[17] E. Calabrese, D. Alonso, and J. Dunkley, Phys. Rev. D 95, 063504 (2017).

[18] A. Lewis and S. Bridle, Phys. Rev. D 66, 103511 (2002).

[19] G. Cabass, A. Melchiorri, and E. Pajer, Phys. Rev. D 93, 083515 (2016).

[20] A. A. Starobinsky, Phys. Lett. 91B, 99 (1980).

[21] J. Garcia-Bellido and D. Roest, Phys. Rev. D 89, 103527 (2014).

[22] R. Easther and H. V. Peiris, J. Cosmol. Astropart. Phys. 09 (2006) 010.

[23] K. Kohri and T. Matsuda, J. Cosmol. Astropart. Phys. 02 (2015) 019.

[24] D. J. Chung, G. Shiu, and M. Trodden, Phys. Rev. D 68, 063501 (2003).

[25] L. Covi, D. H. Lyth, A. Melchiorri, and C. J. Odman, Phys. Rev. D 70, 123521 (2004).
[26] K. Abazajian, K. Kadota, and E. D. Stewart, J. Cosmol. Astropart. Phys. 08 (2005) 008.

[27] M. Joy, E. D. Stewart, J. O. Gong, and H. C. Lee, J. Cosmol. Astropart. Phys. 04 (2005) 012.

[28] G. Cabass, E. Di Valentino, A. Melchiorri, E. Pajer, and J. Silk, Phys. Rev. D 94, 023523 (2016).

[29] E. Di Valentino, S. Gariazzo, M. Gerbino, E. Giusarma, and O. Mena, Phys. Rev. D 93, 083523 (2016).

[30] O. Pisanti, A. Cirillo, S. Esposito, F. Iocco, G. Mangano, G. Miele, and P. D. Serpico, Comput. Phys. Commun. 178, 956 (2008).

[31] F. E. Wietfeldt and G. L. Greene, Rev. Mod. Phys. 83, 1173 (2011).

[32] F. E. Wietfeldt, arXiv:1411.3687.

[33] L. Salvati, L. Pagano, R. Consiglio, and A. Melchiorri, J. Cosmol. Astropart. Phys. 03 (2016) 055.

[34] A. T. Yue, M. S. Dewey, D. M. Gilliam, G. L. Greene, A. B. Laptev, J. S. Nico, W. M. Snow, and F. E. Wietfeldt, Phys. Rev. Lett. 111, 222501 (2013).

[35] A. P. Serebrov et al., Phys. Rev. C 78, 035505 (2008).

[36] R. W. Pattie, Jr. et al., arXiv:1707.01817.

[37] J. B. Muñoz, E. D. Kovetz, A. Raccanelli, M. Kamionkowski, and J. Silk, J. Cosmol. Astropart. Phys. 05 (2017) 032.

[38] T. Sekiguchi, T. Takahashi, H. Tashiro, and S. Yokoyama, arXiv:1705.00405.

[39] K. Kohri, Y. Oyama, T. Sekiguchi, and T. Takahashi, J. Cosmol. Astropart. Phys. 10 (2013) 065.

[40] J. Chluba, R. Khatri, and R. A. Sunyaev, Mon. Not. R. Astron. Soc. 425, 1129 (2012).

[41] J. D. Bowman et al., arXiv:1410.5311.

[42] L. Capparelli, J. Chluba, E. Di Valentino, and A. Melchiorri (to be published).

[43] J. Chluba and R. M. Thomas, Mon. Not. R. Astron. Soc. 412, 748 (2011).

[44] S. Seager, D. D. Sasselov, and D. Scott, Astrophys. J. Suppl. Ser. 128, 407 (2000).

[45] W. Y. Wong, A. Moss, and D. Scott, Mon. Not. R. Astron. Soc. 386, 1023 (2008).

[46] J. Chluba, J. Fung, and E. R. Switzer, Mon. Not. R. Astron. Soc. 423, 3227 (2012). 